\title{
FREQUENCY OF TOOTH LOSS IN DIABETIC SMOKERS
}

1. BDS

Post Graduate Trainee

Department of Dentistry

Fatima Memorial Hospital Lahore

2. MBBS, M.Phil

Assistant Professor

Department of Physiology

Aziz Fatimah Medical and Dental

College, Faisalabad.

3. FCPS

Assistant Professor

Orthopedic Surgeon

DHQ Faisalabad.

4. MBBS, M.Phil

Associate Professor

Department of Physiology

Aziz Fatimah Medical and Dental

College, Faisalabad.

Correspondence Address:

Dr. Shireen Jawad

Department of Physiology

Aziz Fatimah Medical and Dental

College,

Faisalabad.

drshireenjawed@gmail.com

Article received on:

04/02/2019

Accepted for publication:

$12 / 04 / 2019$

\begin{abstract}
Rabia Zia1, Benash Altaf ${ }^{2}$, Zeeshan Ali Khan ${ }^{3}$, Shireen Jawad ${ }^{4}$
ABSTRACT: Edentulism is a condition which is associated with tooth loss. It can be partial or complete edentulism and is the most common oral health issue. It affects subjects of every age group. Multiple factors are believed for causing tooth loss including from poor oral hygiene to systemic diseases. Aim of this study is to highlight the causative factors which are believed to be associated with increased number of tooth loss. Hence, by identifying these factors, preventive techniques could be opted in time in order to prolong the tooth life. Study Design: Cross-sectional study. Setting: Fatimah Memorial Medical and Dental College, LHR. Period: August 20118 to December 2018. Material \& Methods: 100 subjects were recruited by trained dentist after following standard protocols. After approval from ethical committee all the demographic data and relevant information was entered into predesigned proforma. Data was analyzed in SPSS version 21.0. Result: Study showed significant results showing that smoker diabetic subjects have more number of tooth loss. Conclusion: Smoking and diabetes are strong contributory factors for increased number of tooth loss.
\end{abstract}

Key words: Diabetes, Edentulism, Smoking, Oral hygiene

Article Citation: Zia R, Altaf B, Khan ZA, Jawad S. Frequency of Tooth loss in Diabetic smokers. Professional Med J 2019; 26(12):2112-2115.

DOI: 10.29309/TPMJ/2019.26.12.3221

\section{INTRODUCTION}

Tooth loss whether partial or total, is a significant oral health issue globally. Poor oral hygiene and unhealthy habits like smoking, alcohol abuse and systemic diseases like diabetes are triggering factors for this ailment. Diabetes and its associated comorbidities is still challenging in developing and developed countries. In Pakistan prevalence of diabetes mellitus is 11.7 $\%$ being more prevalent in men than in women. ${ }^{1}$ Diabetic subjects are more prone for tooth loss as compared to non-diabetics of their same age group. Increase dental loss among diabetes patients is associated with high incidence and severity of dental plaques and inflamed bleeding gums which results from bacterial growth. These all factors if not managed timely causes damage to the alveolar bone, subsequently leading to tooth loss. ${ }^{2,3}$ It is well documented that one of the crucial cause for developing diabetes is smoking. ${ }^{4}$

According to the data of world health organization, incidence of smoking is increasing at a break neck pace. Our young generation use to smoke in an illusion of looking cool, health care professionals smoke because they feel themselves alert and active to perform their hectic routine smoothly while others think that it distracts them from surroundings that make them upset. ${ }^{5}$ Smokers ultimately have to suffer from multiple diseases in their life like diabetes, hypertension, acute coronary syndrome, chronic obstructive lung diseases and even different types of carcinomas. Smoking not only affects systemic organ but also involves nasal and oral hazards. It includes from some benign daily problems to life threatening conditions, like bad breath, tooth discoloration, taste and smell alteration, poor oral wound healing, periodontal disease, oral mucosal lesions such as smoker's melanosis and smoker's palate. On the other hand, malignant conditions may involve carcinomas oral cavity which comprised $92 \%$ deaths of all the cancers developed in the result of smoking.

Other carcinomas developed as result of smoking 
include carcinoma pharynx, larynx and lungs. Smoking done in any of the form first lead to poor oral hygiene which ultimately involves changes in gums like swelling, bleeding and ultimately pocket formation in gums. These all features when present simultaneously are considered as a disease known as periodontitis. It can loosen up the teeth from gums that might eventually be fallen out or taken out by the dentists. ${ }^{6,7}$ Smoking along with diabetes also results in ineffective treatment for gums causing edentulism. In America smoking is the major cause for periodontitis, poor gum healing with treatment and ultimately leading to tooth loss..$^{8,9}$ Smokers are 30-40\% more prone to develop type 2 diabetes than nonsmokers. People with diabetes who smoke are more likely to have trouble with insulin dosing which ultimately lead to systemic as well as oral health complications than nonsmokers. ${ }^{10,11}$ Positive association of cigarette smoking with diabetes is well documented. It is believed to cause diabetes as it increases oxidative stress and augments the process of inflammation along with directly damaging the $\beta$-cell function. These all factors play a vital role in the pathogenesis of diabetes. ${ }^{4}$

So as a result, in order to promote oral health and function, understanding the causes for tooth loss can enable the development of preventive policies that can also have favorable effect on associated morbidities. Aim of the study is to check the frequency of tooth loss among diabetes nonsmoker and diabetes smokers. Regular dental care and good oral hygiene practices have been demonstrated to reduce the incidence of dental loss; however, there is inadequate emphasis on prevention and treatment for dental problems among medical providers caring for patients with diabetes.

\section{METHODOLOGY}

100 subjects were recruited from Fatimah Memorial Hospital (dental section) Lahore after a complete oral examination by trained and calibrated dentist following standard protocols. Information was gained about participants' smoking habits with help of standardized selfadministered questionnaire which was completed and handed over to dentist during a visit. We enrolled smoker subjects with tooth loss who were having diabetes or no diabetes. Then we categorized subject on the basis of diabetes into diabetes smoker, diabetes nonsmokers and non-diabetes smoker, non-diabetes nonsmoker. Descriptive were taken in form of frequencies and percentages. Tooth loss among studied subjects was compared using the chi-square. Value less than0.05 was taken as significant.

\section{RESULTS}

Current study comprised of 100 subjected, recruited from dental section OPD of Fatimah Memorial Medical and Dental College, LHR. Out of these 100 subjects 64 (64\%) were male and $36(36 \%)$ were female (Figure-1). Mean age of recruited subjects was $40 \pm 14.2 .56(56 \%)$ of the studied population was smoker and 44 (44\%) was nonsmoker. Out of this, 89 (89\%) of the studied subjects were diabetics while $11(11 \%)$ of the subjects were non diabetics. Chi-square test was applied to see the frequencies and percentages. Study showed a significant results $(P=0.000 *)$ for tooth loss in diabetic smoker subjects (Table-I).

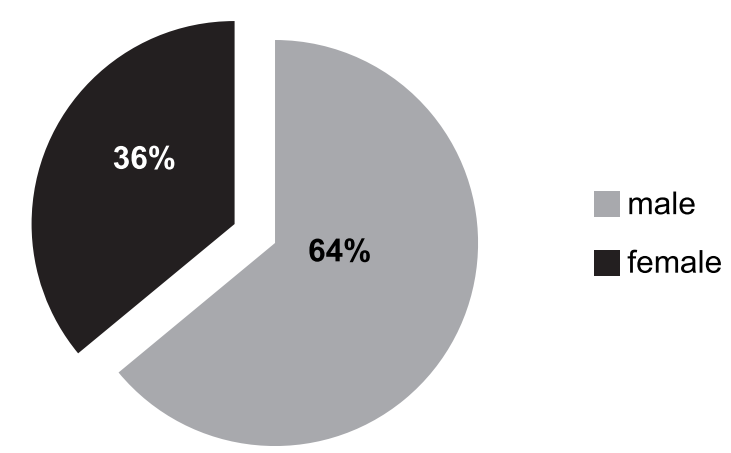

Figure-1. Distribution of gender among the studied population.

\begin{tabular}{|c|c|c|c|}
\hline & \multicolumn{2}{|c|}{ No of Tooth Loss } & \multirow[b]{2}{*}{ P-Value } \\
\hline & $\begin{array}{c}1 \text { to } 4 \\
N=(\%)\end{array}$ & $\begin{array}{c}\text { More than } 4 \\
\mathrm{~N}=(\%)\end{array}$ & \\
\hline Diabetics & $0(0 \%)$ & $89(89 \%)$ & \multirow{2}{*}{$0.000 *$} \\
\hline Non-diabetic & $10(10 \%)$ & $1(1 \%)$ & \\
\hline
\end{tabular}




\begin{tabular}{|l|c|c|c|}
\hline & \multicolumn{2}{|c|}{ No. of Tooth Loss } & \multirow{2}{*}{ P-Value } \\
\cline { 1 - 3 } & $\begin{array}{c}\mathbf{1} \text { to 4 } \\
\mathbf{n}(\%)\end{array}$ & $\begin{array}{c}\text { More than 4 } \\
\mathbf{n}(\%)\end{array}$ & \\
\hline Smoker & $4(40 \%)$ & $52(57.8 \%)$ & \multirow{2}{*}{0.229} \\
\hline Non smoker & $6(60 \%)$ & $38(42.2 \%)$ & \\
\hline
\end{tabular}

Table-II. Tooth loss among smokers and nonsmokers

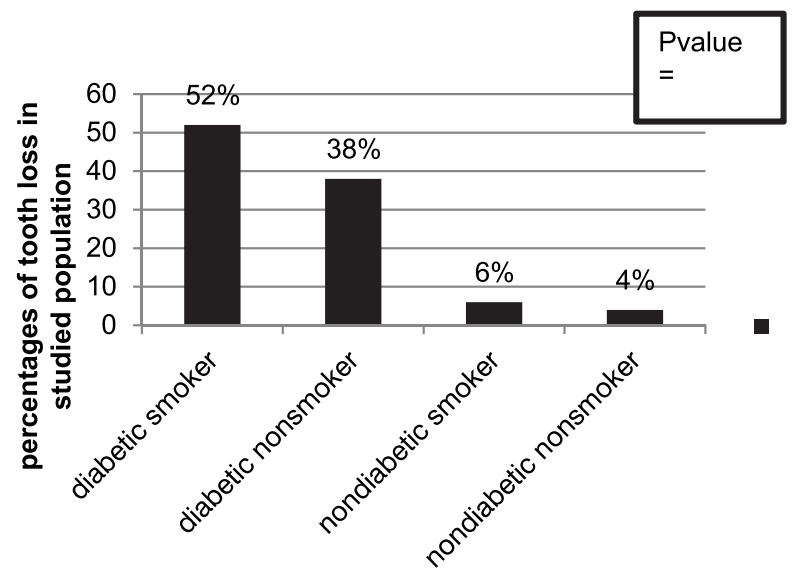

Figure-2: Percentages of tooth loss among studied subjects

\section{DISCUSSION}

Tooth loss is a common oral health issue. Children as well as adults are affected by this condition. Most of the affected subjects are those who follow poor oral hygiene and unhealthy habits like smoking and alcohol abuse etc. Other systemic diseases especially diabetes is believed to play a major role for tooth loss. Need for highlighting tooth loss globally is its concern with proper chewing and proper absorption of diet which otherwise would lead to malnourishment irrespective of the age. Uncontrolled diabetes is believed to play a key role for establishing macrovascular as well as microvascular diseases involving kidneys, eyes, nerves, heart and brain. Substantial amount of data is available concerning diabetes systemic effect and has resulted in particular guidelines for preventing and managing the complications like regular eyes monitoring, foot and wound care etc. Contrary to that, data addressing effect of diabetes on oral health is still not sufficient by our health providers. Tooth loss resulting from periodontal disease is more prevalent in smokers and diabetes but still it has not received similar importance as given to other complications of diabetes. There is still lack of data showing fruitful impact on required outcomes achieved with aggressive dental health care in diabetics patients. ${ }^{3,11}$ In this study, we assessed oral health status of a studied sample of diabetes patient. Current study showed that diabetic subjects have more percentage of tooth loss $89(89 \%)$ as compared to non-diabetics $10(10 \%)$ showing significant results of tooth loss among diabetics $\left(P\right.$ value $\left.0.000^{*}\right)$. This was in accordance to the study of Kenneth E. Izuora who also found that diabetic subject are more prone to lose their tooth compared to their age fellows.

We also found that smoking is also a contributory factor for plaque formation and periodontitis. In our study we found that smokers have increased tendency to lose more number of teeth i.e 52 (57.8\%) as compared to nonsmokers 38(42.2\%) Table-II. But the results were not found to be statistically significant. It may be attributed to small sample size. Izoura also found non-significant results which were similar to ours. ${ }^{3}$ Current study showed increase number of tooth loss in diabetic smokers 52(52\%) when compared with diabetic nonsmokers 38 (38\%) Figure-2. Number of tooth loss was statistically significant in diabetic smoker subjects $\left(P=0.000^{*}\right)$. Similar to our results, Javed et al made comparison of Periodontal disease in smokers and non-smokers with T2DM and found that, periodontal inflammation disease is more common and worst in smokers than non-smoker diabetic subjects. ${ }^{12}$ This relationship can be explained that smoking not only causes diabetes by directly damaging the beta cell ${ }^{13,14}$ but it also lead to poor oral hygiene and wound healing. ${ }^{13}$

This study will open new horizons in the oral health of diabetic smoker subjects. This will not only make diabetic subjects more cautious to take care of their oral hygiene but will also improve nutritional outcomes. Further researches are needed on broader scale in order to put emphasis on oral hygiene in diabetic subjects rather than only focusing on systemic complications of diabetes which is practiced by our many health care providers. Moreover, strong association of diabetes and smoking and then its consequences on oral health should be elucidated on broader scale too so that preventive measures could 
be opted in time before reaching the ultimate consequences.

\section{CONCLUSION}

Our verdicts highlight the disease load in diabetes smoker subjects that results from poor dental care. There is a significant and noteworthy association of dental loss in diabetic smokers. Our findings also recommend that good dental hygiene, with strong emphasis on regular dental flossing, and proper dental clinic visits may have a significant effect for preventing tooth loss in diabetic smokers.

Copyright@ 12 Apr, 2019.

\section{REFERENCES}

1. Meo SA, Zia I, Bukhari IA, Arain SA. Type 2 diabetes mellitus in Pakistan: Current prevalence and future forecast. J Pak Med Assoc. 2016; 66 (12):1637-1642.

2. Martin JA, Page RC, Loeb CF, Levi PA Jr. Tooth loss in 776 treated periodontal patients. J Periodontol 2010; 81 (2):244-50.

3. Kenneth E. Izuora, Echezona E. Ezeanolue, Dental loss among ambulatory patients with diabetes, J Clin Transl Endocrinol.2016;4:28-31.

4. Chang SA Smoking and type 2 diabetes mellitus. Diabetes Metab J. 2012; 36 (6):399-403. doi: 10.4093/ dmj.2012.36.6.399.

5. Raoul A. Walsh, Encouraging people to stop smoking, Department of mental health and substance dependence world health organization Geneva. WHO/MSD/MDP/01.4

6. Centersfordiseasecontroland prevention. Periodontal Disease [last updated 2015 Mar 10; accessed 2018 Mar 22.
7. National Institute of Dental and Craniofacial Research. Periodontal (gum) disease: Causes, symptoms, and treatments [accessed 2018 Mar 22].

8. Eke PI, Dye BA, Wei L, et al. Prevalence of periodontitis in adults in the United States: 2009 and 2010. Journal of Dental Research 2012; 91(10):914-20.

9. U.S. Department of Health and Human Services. The Health Consequences of Smoking-50 Years of Progress: A Report of the Surgeon General. Atlanta: U.S. Department of health and human services, centers for disease control and prevention, national center for chronic disease prevention and health promotion, Office on Smoking and Health, 2014.

10. $\mathrm{Xu} \mathrm{H}$, Wang $\mathrm{Q}$ etal, Environmental Science and Pollution Research In type 2 diabetes induced by cigarette smoking, activation of p38 MAPK is involved in pancreatic $\beta$-cell apoptosis. Environ Sci Pollut Res Int. 2018; 25(10): 9817-9827. doi: 10.1007/s11356-0181337-3.

11. Tasdemir Z, Alkan BA. Knowledge of medical doctors in Turkey about the relationship between periodontal disease and systemic health. Braz Oral Res 2015; 29(1):55.

12. Javed F, Al-Kheraif AA, Salazar-Lazo K, YanezFontenla V, Aldosary KM, Alshehri M, et al. Periodontal inflammatory conditions among smokers and never smokers with and without type 2 diabetes mellitus. J Periodontol 2015; 86(7):839-46.

13. Wang $\mathrm{C}$, Wang $\mathrm{Y}, \mathrm{Wu} \mathrm{J}$ et al. Journal of diabetes research. Current smoking dose-dependently associated with decreased $\beta$-Cell function in Chinese men without diabetes, 2015, Article ID 841768, 9

14. Li T, Ni L, Zhao Z, Liu X, etal. Melatonin attenuates smoking-induced hyperglycemia via preserving insulin secretion and hepatic glycogen synthesis in rats. J Pineal Res 2018; 64(4). e12475. doi: 10.1111/ jpi.12475.

\begin{tabular}{|c|c|c|}
\hline \multicolumn{3}{|c}{ AUTHORSHIP AND CONTRIBUTION DECLARATION } \\
\hline Sr. \# & Author(s) Full Name & \multicolumn{1}{|c|}{ Contribution to the paper } \\
\hline 1 & Rabia Zia & $\begin{array}{l}\text { Contributed to acquisition, She is the guarantor of } \\
\text { this work and as such has full access to the data to } \\
\text { all the data in the study and takes responsibility for } \\
\text { the integrity of the data and the accuracy of the data } \\
\text { analysis. } \\
\text { Contributed to study design, wrote the manuscript } \\
\text { and reviewed references. She also contributed to data } \\
\text { analysis, interpretation, editing and formatting the } \\
\text { manuscript. Reviewed and approved the manuscript. } \\
\text { Contributed in editing and formatting the manuscirpt. } \\
\text { Reviewed and approved the manuscript. } \\
\text { Contributed to study design, helped in statistical } \\
\text { analysis and manuscript writing. Revising it critically } \\
\text { for important intellectual content. }\end{array}$ \\
\hline 3 & Benash Altaf & Zeeshan Ali Khan \\
\hline 4
\end{tabular}

